\title{
The ECH front steering launcher for the ITER upper port
}

\author{
R. Chavan, M. Henderson, R. Bertizzolo, J.-D. Landis, F. Sanchez and H. Shidara \\ CRPP - EPFL, Association EURATOM - Confédération Suisse, 1015 Lausanne, Switzerland
}

The purpose of the ITER electron cyclotron resonance heating (ECRH) upper port antenna will be to drive current locally inside the island which forms on the $q=3 / 2$ or 2 rational magnetic flux surfaces in order to stabilize neoclassical tearing modes (NTMs). The launcher should be capable of steering the beam deposition location across the resonance surface over the range in which the $\mathrm{q}=3 / 2$ and 2 surfaces are found, for the various plasma equilibria susceptible to the onset of NTMs. ITER's reference design uses a front steering (FS) concept, with the moveable mirror close to the plasma. Two separate mirrors are used to decouple the focussing and steering aspects resulting in an optimized folded beam path, and an improved performance over the alternative remote steering concept. In addition the steering range can be extended offering a synergetic design with the equatorial launcher and the prospect for an enhanced ECH physics programme on ITER.

The FS launcher is capable of steering eight 2MW beams via two sets of steering mirrors out of each of the four allocated upper port plugs. Among the relevant mechanical design aspects are the systematic reliance on frictionless and backlash free mechanical movements based on the compliant deformation of structural components to avoid the in vessel tribological difficulties [1]. An inert gas pressure controlled bellows system provides accurate angular positioning of the steering mirror. Integrated design solutions provide sufficient neutron shielding within the available space. The antenna is designed fail-safe to provide full bandwidth beam steering capabilities during the twenty years lifetime of ITER. Details of the FS launcher design relating to mm-wave, thermohydraulic, electromagnetic, neutron shielding and structural aspects and possible options regarding the integration of launcher components into the port plug are discussed.

The scope of the mm-wave engineering and design work on the FS launcher system is detailed, which includes the routing of the mm-wave guides, starting at the inlet gatevalves near the closure plate through the port plug and neutron shielding structures. The particular key design features developed in response to the functional and physics requirements are explained and assessed.

[1] J.-D. Landis et al., this conference. 